\title{
Use of Drone based Lidar technology at Olympic Dam Mine and initial Technical Applications
}

\author{
D Woolmer BHP Olympic Dam, Australia
}

E Jones BHP Olympic Dam, Australia

J Taylor BHP Olympic Dam, Australia

C Baylis University of Melbourne, Australia

D Kewe James Cook University, Australia

\begin{abstract}
Olympic Dam Mine is a copper, uranium, gold and silver mine located $560 \mathrm{~km}$ north of Adelaide, South Australia. The orebody sits beneath approximately 300 metres of a surface, sedimentary sequence and extends to over 2,000 metres in some of the southern areas. The mining method utilized is sub-level open stoping (SLOS) with variable stope sizes and geometries depending on localised mineralization and ground conditions. Obtaining accurate stope survey data is a key part of managing material risks and planning inputs at Olympic Dam. Conventional stope survey methods are subject to issues such as resources required and variable data quality, leading to delays in the production cycle. This process has been improved by the implementation of Emesent's Hovermap drone autonomy and mobile LiDAR mapping payload. This paper provides a 12-month review describing how Olympic Dam has implemented the technology. It explains the process of training underground personnel to become skilled pilots and flights and scans are scheduled into operations. The results of using this new technology are then compared to traditional survey methods in regards to implementation, output quality and safety. The paper then discusses in detail the applications that the Mine Technical Services Department (Survey, Geotechnical, Geology and Mine Scheduling) are using the data to improve knowledge of the mine and implement efficiencies. The additional data provided has been used by the Technical Services team to refine structural models and actively analyse future stope designs in new mining areas. The introduction of drones, big-data and point-clouds have all been challenges as they are outside the norm for the operation. However, once overcome the benefits from this type of technology and the data it generates is proving to be invaluable for improving safety, time efficiencies and understanding of the rock mass that is feeding into the downstream mining processes.
\end{abstract}

\section{Introduction}

The Olympic Dam Mine is located in northern South Australia, approximately $560 \mathrm{~km}$ north of Adelaide. The Olympic Dam deposit itself is a poly-metallic deposit containing copper, uranium, gold and silver. The mine has been operated by BHP Group Ltd since 2005, following the takeover of Western Mining Corporation (WMC), who discovered and operated the mine since its opening in 1988.

Olympic Dam utilises the Sub-Level Open Stoping (SLOS) Method and is one of the largest stoping mines in the world. Stope size and geometry is variable and dependent upon localized mineralisation and geotechnical characteristics, particularly in the Southern Mining Area (SMA), where operations have recently commenced. Ore is processed and refined on site to produce copper cathode, uranium oxide concentrate along with minor quantities of gold and silver bullion. 


\section{Review of previous stope survey technology and methods}

The process for measuring underground voids prior to the implementation of drone technology has been the Cavity Monitoring System (CMS) and Cavity Auto-scanning Laser System (C-ALS) systems. Both these systems have been the industry standard for void measurement and have been used globally for a number of years, however the use of this technology at Olympic Dam has highlighted some operational constraints:

1. To conduct a CMS survey personnel are required to be in close proximity to the void due to the range of the equipment. This proximity is a risk to personnel due to hazards such as Fall from Height, interaction with falling stope material and Fall of Ground from stressed ground conditions.

2. The use of CMS scanners is relatively resource intensive, requiring personnel and equipment, necessitating scheduling to reduce interactions and maintain output.

3. Due to the fixed nature of the equipment, 'shadowing' can occur on scans, where the scan cannot reach all areas of the stope due to the geometry of the stope or undulations in the stope walls due to overbreak or underbreak. This is prevalent in Olympic Dam due to the drawbell design of the stopes where the base of the stope tapers towards the drawpoints. Due to safety concerns, CMS scans are not allowed at the drawpoint level, meaning that CMS scans must be taken from the level above. This lack of clarity of the base of stopes has resulted in incomplete scans, leading to production delays to obtain a second scan from a second location or loss of material as adjacent stopes are designed with a rock pillar to account for potential unconsolidated material in the original stope; and

4. C-ALS surveys are typically taken when access is severely restricted. For the use of C-ALS survey a hole into the target void must be present, which requires the use of a Simba drill rig to drill the hole, while also being required to clean out any previous holes for the C-ALS to take place. All of these resources are required for daily mining operations and using these units for surveys reduces productivity and requires prior scheduling to eliminate any potential interactions and reduce cycle downtime to as low as practicable.

\section{Operational impact}

Due to the aforementioned operational requirements to conduct stope surveys, these surveys are limited to post production scans or events of variance from original designs. As a result the production of a stope is measured by tracking material movements. Inputs are calculated from original firing designs and outputs are calculated using standardised bucket volumes multiplied by the specific gravity to obtain a tonnage, which is then multiplied by the number of buckets removed. These figures are then used to derive a stock level, which is used in monthly production scheduling and planning. Due to the number of assumptions made in this process, variance is common as a result of underbreak or overbreak and non-conformity of buckets extracted from a stope, due to fragmentation and operator variance. These variances can lead to a significant difference between calculated and actual stope stock levels, which can impact production output. These variances can also lead to significant stock adjustments post reconciliation when production output from factored calculations is compared to a known measure point located at the Olympic Dam Concentrator.

In late-2018, multiple drone platforms were considered for use at Olympic Dam to improve the volume, quality and overall safety of the void survey process. Upon review the Hovermap Lidar system from Emesent was chosen due its specialised design for use in the underground mining environment. 


\section{$4 \quad$ Introduction of Hovermap mobile LiDAR system to Olympic Dam}

\subsection{Scanning Hardware overview}

The Hovermap Lidar mapping system used at Olympic Dam was supplied by Emesent Pty Ltd, previously part of the CSIRO's 'Data 61' Project. The Hovermap system consists of three elements; a 16 channel Velodyne scanner, Inertial Measurement Unit (IMU) and on-board computer system (Jones et al. 2019). This system is a demountable system weighing approximately $2.5 \mathrm{~kg}$ and can be mounted onto most Unmanned Aerial Vehicles (i.e. Drones). The power source is derived directly from the drones battery system or can be powered by external batteries.

The Velodyne scanner produces up to 300,000 points per second allowing for high-resolution scans to be produced. This high point cloud density combined with on-board processing allows for the scanner to conduct Simultaneous Location and Mapping (SLAM), enabling the system to monitor its position relative to its surroundings in real time. The system also includes a Virtual Ellipsoidal Shield (VESH), a 3-dimensional customisable barrier around the drone, preventing collision with the environment, which has been a key feature for use in confined areas associated with underground mining.

The files produced from the Hovermap scanner are processed using vendor developed software, producing point cloud data in .laz file format, which can be converted into varying file types for integration into survey and mining specific design software.

\subsection{Project scoping and preparation}

Prior to receiving the Hovermap system an initial project briefing was conducted to discuss how the system would be integrated into the Olympic Dam operations. The first discussion point centred on personnel responsible for conducting drone scanning flights, as this system would impact on multiple departments and processes, creating a large pool of candidates. After discussions it was decided that the pilot team would be derived from the Underground Production Team. The reasons for this decision were:

1. Underground executions personnel work on a continuous roster, which would allow for continual coverage of drone pilots;

2. Underground personnel are experienced in working in the underground environment and are able to observe and rectify any hazards. Underground Production personnel in particular are experienced in working around stope voids and identifying hazards; and

3. Training Underground personnel in the use of this system will upskill members of the team allowing those individuals to assist in drone based work required on the surface.

In regards to the individuals no pre-requisites were deemed mandatory due to the training the individuals would receive prior to commencing the role. Individuals were assessed based on complimentary skills such as hand-eye coordination, attention to detail, technological acumen and underground experience. Previous experience in flying drones was deemed advantageous but non-essential due to the aforementioned training.

Once the criteria was established, the management team engaged with the mine supervisors to nominate personnel for this role. The resulting group were then narrowed down to nine persons, spread across the four underground crews. 


\section{Implementation}

\subsection{Initial training}

The first iteration of the Hovermap system was delivered to Olympic Dam in February 2019, consisting of three components; one DJI Matrice 600 (M600) Drone, vertically mounted Hovermap Lidar and associated processing hardware.

The equipment was accompanied by two representatives from Emesent for initial training in the use of the equipment. This training consisted of surface operation of smaller drones, progressing to operation of the M600 drone and finally operation of both drone types in controlled underground settings. An example of this training and equipment is shown in Figure 1:

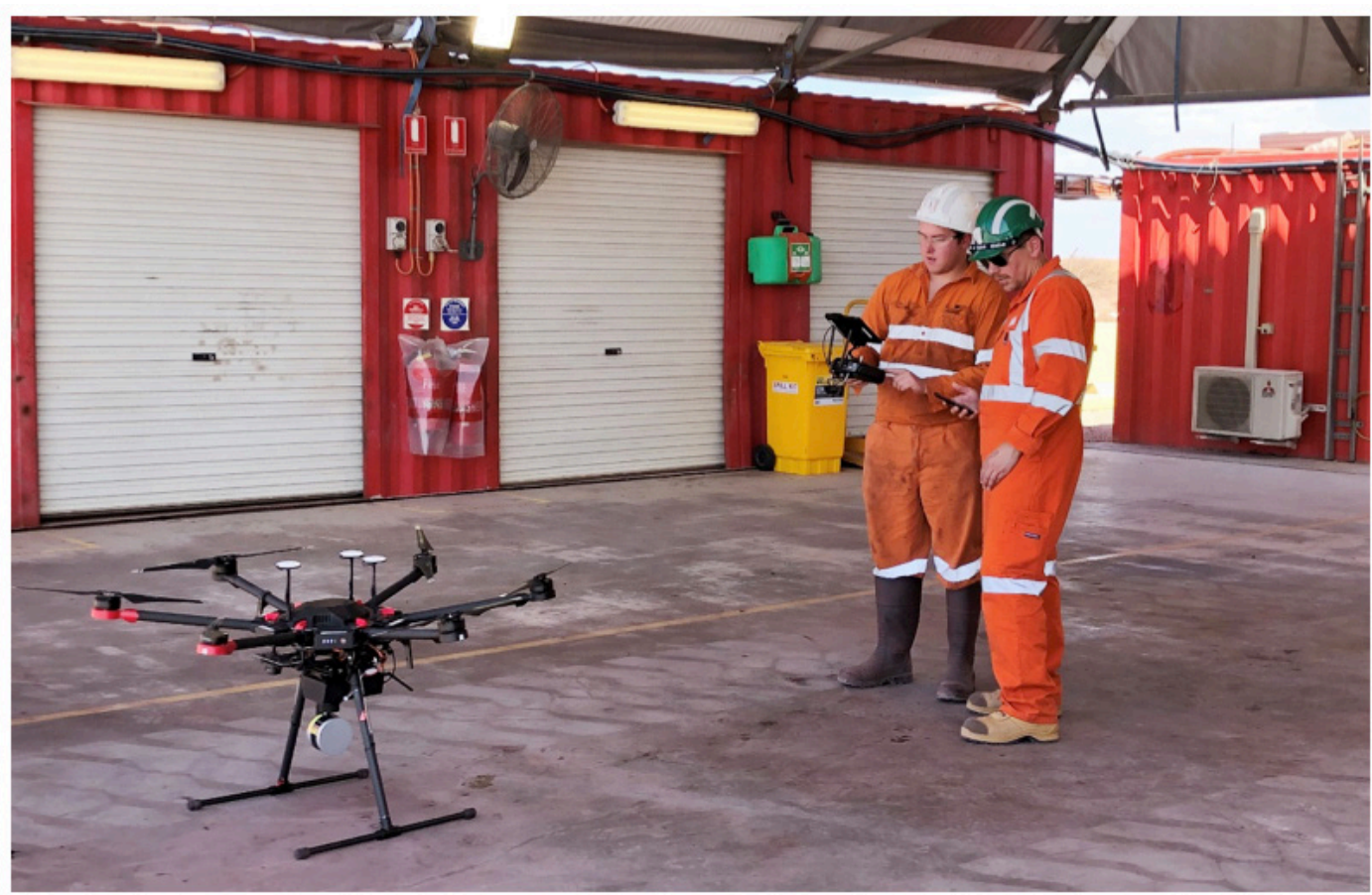

Figure 1 Initial training with Hovermap system

After eight days of training across both operational crews, Olympic Dam were approved to use the Hovermap system by Emesent. The pilot team were accompanied on the training by the Production Analysis and Improvement Team and the Olympic Dam Mining Team to observe the training conducted and use the training as the basis of training documentation in consultation with Emesent.

Post-delivery all Olympic Dam pilots were placed on the Remote Pilots License (RePL) training course to obtain their multi-rotor drone licenses for drones up to $7 \mathrm{~kg}$. This training was conducted by a Civil Aviation Safety Authority (CASA) certified training supplier, consisting of theory training and five hours of practical flying to show competency in drone operation. This course was conducted over five days and all pilots received their licenses thereafter. In August and December 2019 further operators at OD participated in the RePL course to expand the drone pilot team. Olympic Dam now has 20 certified drone pilots spread across four operational crews. 


\subsection{Initial use of drone mounted system for scanning stope voids}

Implementation of the drone and LiDAR system in the initial stages was quite slow, with low output and a high number of aborted scans. A root cause analysis was conducted on this issue and two causes were isolated for the low performance;

- Poor scheduling of tasks; and

- A lack of understanding regarding the systems capabilities.

In the early stages scans were conducted on an ad-hoc basis where sites were chosen on a daily basis. This meant that sites were not inspected prior to requests being made. On consultation with the underground leadership team there was a prevalent assumption that the drone could access tight areas and operators could operate the machinery from a large distance. This meant that sites chosen had not had the required clean up necessary to remove obstacles that would interact with the VESH collision system preventing access.

To address these issues a robust scheduling and planning process was established, using the current setup process for CMS scans as a point of reference. Drone scans were scheduled a week prior as part of the eight-day scheduling process for scoping by a member of the pilot team. If the site met the requirements for a flight, the scan would be conducted during that week. If site setup works such as clean-ups and ventilation were required, these works would be scheduled into the following eightday schedule along with the requested flight. This process was managed by the Short-term Scheduling team in conjunction with the Mine Production team. Once this process had been established, a marked increase in completed scans was observed with fewer aborted scans, many of those related to unforeseen operational interactions not covered in the short term schedule. Many of these aborted scans were completed within 48 hours of the initial scheduled scan.

Output tracking of the drone and Lidar system has shown continued growth during 2019, with July the outlier, which was due to maintenance conducted on the drone during that month. The number of scans reaches a steady state rate from September with an average of 23 scans conducted per month during the period (Figure 2).

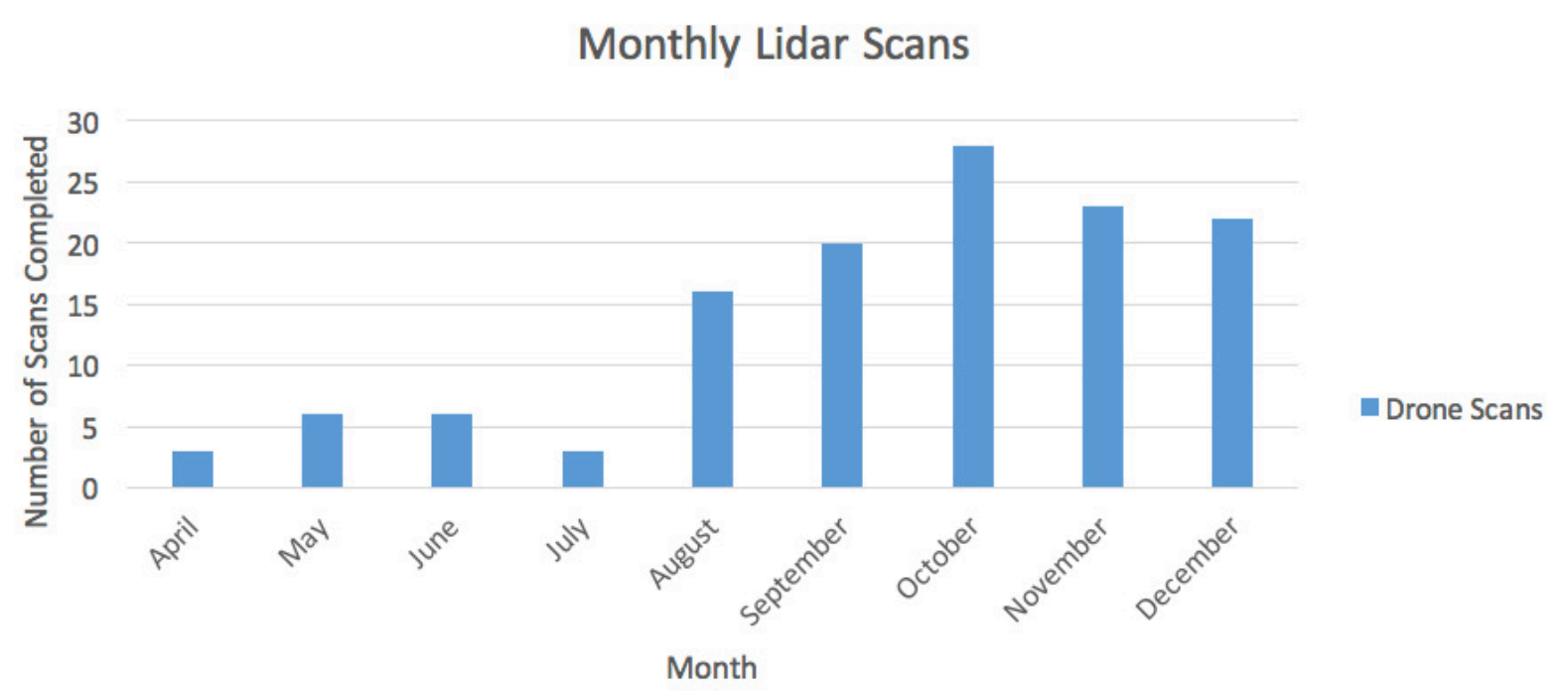

Figure 2 Summary of the number of drone scans completed during 2019

The majority of scans have been taken at the drawpoint and crown levels of the stope. This has been due to the requirements of the scanner to monitor stock levels, interim blast packet performance and stope closeout, which have primary access through these levels. The use of the drone scanner in particular has markedly increased the quality of scans due to the location, resulting in fewer shadowing issues. There have been two events where a CMS has been rejected due to shadowing, followed directly by a drone 
scan at the drawpoint level. This has allowed for direct comparison of the two systems and has shown the improved quality of the drone and Lidar system.

The average scan time measured from 138 scans is 7 minutes and 14 seconds, with an increase in length over time, as personnel have become more familiar with the system and its capabilities, a development which has increased the density of points. The ease of set up of the drone and scanner has enabled multiple scans to be taken during a single shift. Daily output has been increasing in line with monthly output, with daily peak scan output of six scans conducted during December.

Personnel safety has markedly improved with implementation of the system based on increased standoff from the stope void, with personnel based at least 20 metres from the stope void during scanning. It is this increased standoff distance which has allowed drawpoint scans to take place, a process which as mentioned prior was not achievable using previous scanning methods.

In summary the implementation of the Hovermap system has had a definitive impact upon operational and technical processes at Olympic Dam. The systems ease of use has allowed multiple scans to be taken during a shift, increasing data availability particularly of interim stope statuses, allowing greater clarity in stope scheduling. The ability to operate away from the void has allowed scans to be taken of previously inaccessible areas, allowing for greater control of stope stability. The use of a mobile scanner coupled with the ability to safely scan at drawpoint levels has virtually eliminated the issue of shadowing in scans.

The availability of high quality scans comprising of up to 90 million data points available to all technical personnel has enabled data to be used in the modelling and design process, which is expanded upon in Section 7 - Application within the Planning and Technical Departments.

This has resulted in significant growth of data volume and quality compared to traditional scanning methods, data which has been used by technical and operational departments to refine and improve their processes.

As the understanding of the hardware and applications of the scan data has improved, Non-drone mounted configurations for the Hovermap scanner have been utilised.

\section{Platforms used for data acquisition at Olympic Dam}

Post-delivery of the drone and LiDAR system it was noted that the LiDAR scanner could be detached from the drone and used in non-drone configurations. Analysis of non-drone configurations was undertaken to find applications for the LiDAR where the drone was not required.

\subsection{Vehicle mounted scanning}

The use of the drone mounted system at a drawpoint level while an improvement in safety, still required personnel to stand in front of the drawpoint to maintain line of sight. It was identified that the underground loaders in tele remote mode would be a suitable vehicle to carry the scanner, whilst completely removing personnel from the area. This configuration also eliminated the risk of drone damage due to striking by falling material by removing the drone entirely from the process.

For this configuration to be utilised a suitable mounting system would be required that meets two conditions; the mount must be detachable to enable the scanner to be used on multiple units with quick transfer, and secondly the mount must be able to absorb machine based vibration, maintaining scan quality and protect the system from vibration related damage to precise components.

To meet these requirements a mount was developed based on the mount used to attach the scanner onto the drone. This mount was attached to a steel plate with rubber tubing between each mounting point to reduce vibration. The mount attached to the vehicle using four neodymium magnets, with a combined force of $60 \mathrm{~kg}$. The intended use of the mount was on remote operated loaders for the purpose of scanning stopes at the drawpoint level, while maintaining safe operating distance for operators. 
Once the system was fabricated, testing was conducted on a trial stope using a remotely operated loader with three parameters; the ease to mount and remove the system, the amount of vibration observed during the scan and finally the quality of the subsequent scan versus the traditional drone configuration. A loader trial was chosen over a vehicle test as a loader produces higher levels of vibration during operation and hence would be a more effective test of the systems capabilities. Photographs of the system both prior to and during the test are shown in Figure 3 and Figure 4.

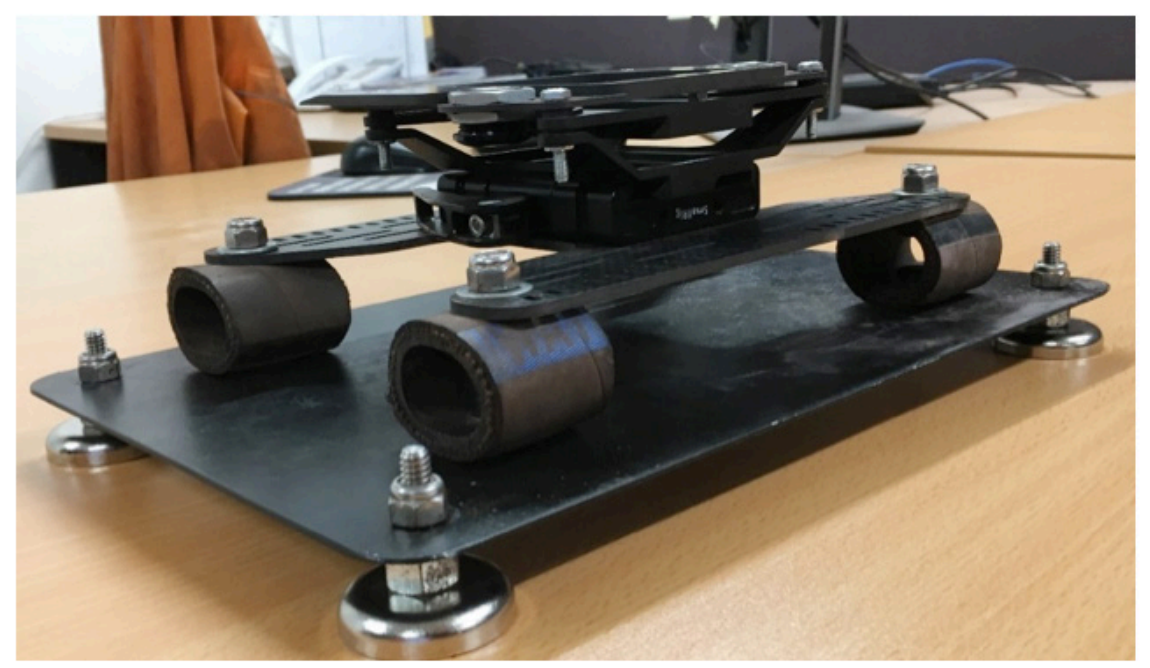

Figure 3 Side view of Hovermap vehicle mount

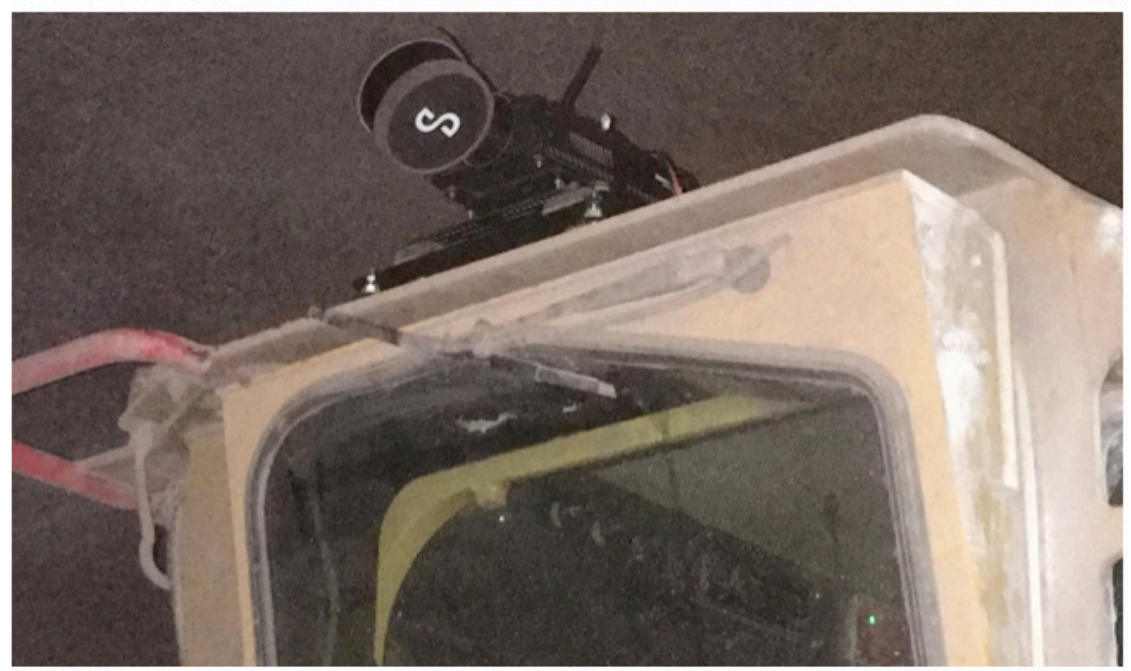

\section{Figure 4 View of the vehicle mounted system attached to cab of a tele-remote loader}

The results of scan showed a scan time of 7 minutes 30 seconds, which is in line with the standard drone configuration. On-board data was sent to the manufacturer for analysis which showed vibration within acceptable limits, with only minor spikes associated with extreme variations in the road surface.

The processed scan showed an operational range of $60 \mathrm{~m}$, which again is in-line with the drone configuration and expected sensor range for underground environments experienced at Olympic Dam. This operational range allowed for the majority of the stope void to be surveyed, with only part of the crown raise not scanned. The density of the points also showed fine details in the stope crown as shown in Figure 5. After the trial this system was passed into general operation with no adjustments. 


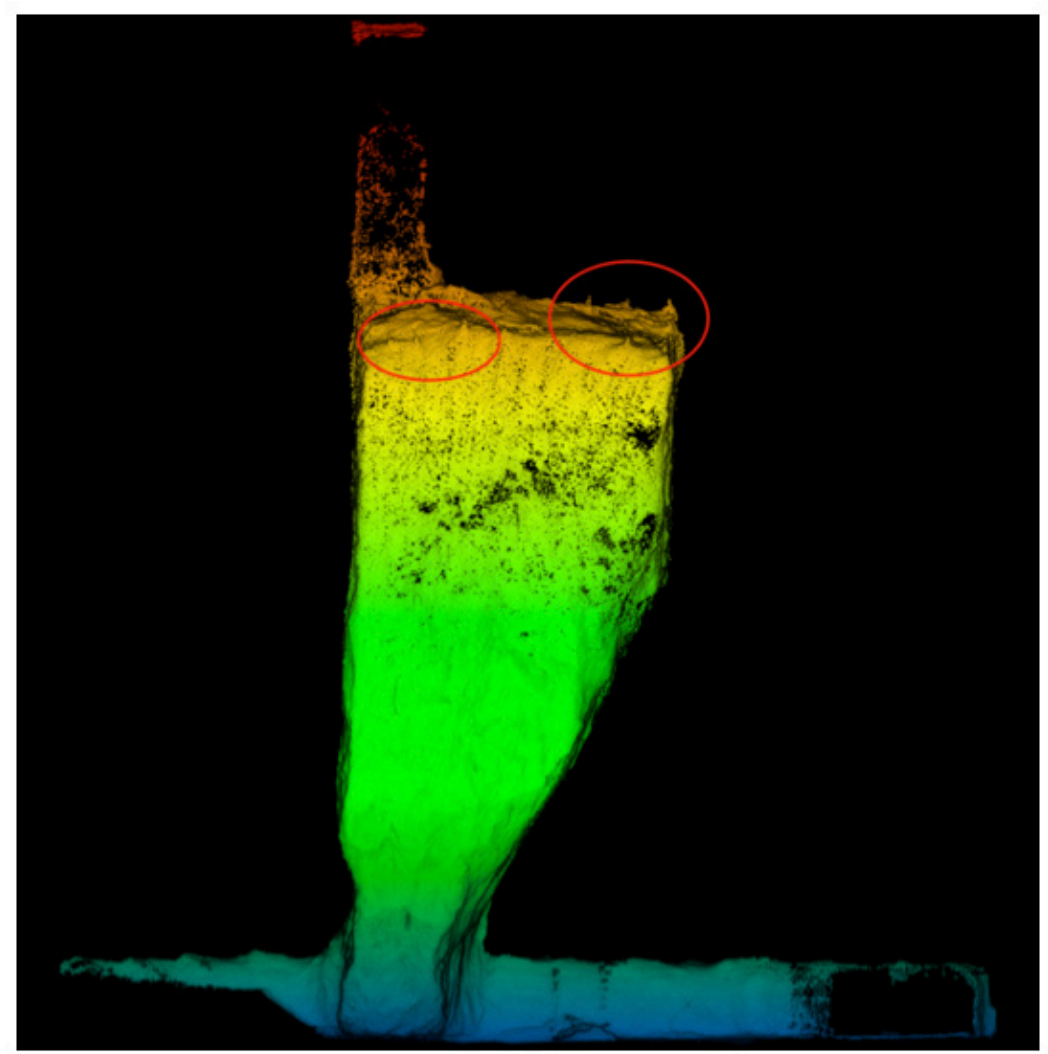

\section{Figure 5 Stope scan using loader mounted scanner configuration}

This system has proved highly successful in the post production stages of the stope, highlighting areas of overbreak in the base of stope and also any ore material remaining in the stope, which can be removed prior to backfilling.

\subsection{Crane mounted scanning}

In November 2019 the Lidar scanner and vehicle mount were used to survey a former hoist shaft, currently used as a ventilation raise, to measure potential deformation in the concrete lining. A hazard zone has been established around the shaft, meaning direct access to the shaft was not possible and wind velocities in excess of $14 \mathrm{~m} / \mathrm{s}$ prohibited use of the drone to conduct the scan.

To enable the scan to take place whilst maintaining personnel safety, the Lidar scanner was attached to a Franna crane (Figure 6). By using the extension capability of the cranes boom, access to the shaft was enabled whilst maintain safe distance from the shaft. And using the operational range of the Lidar scanner the concrete lining was able to be scanned whilst keeping the scanner at a shallow depth, where the impact of the air velocity would be minimal.

The scanner was lowered to a depth of $10 \mathrm{~m}$ below the surface and scanning was conducted for 11 minutes before being safely removed from the shaft (Figure 7). The results from the shaft stability assessment are outlined further below. 


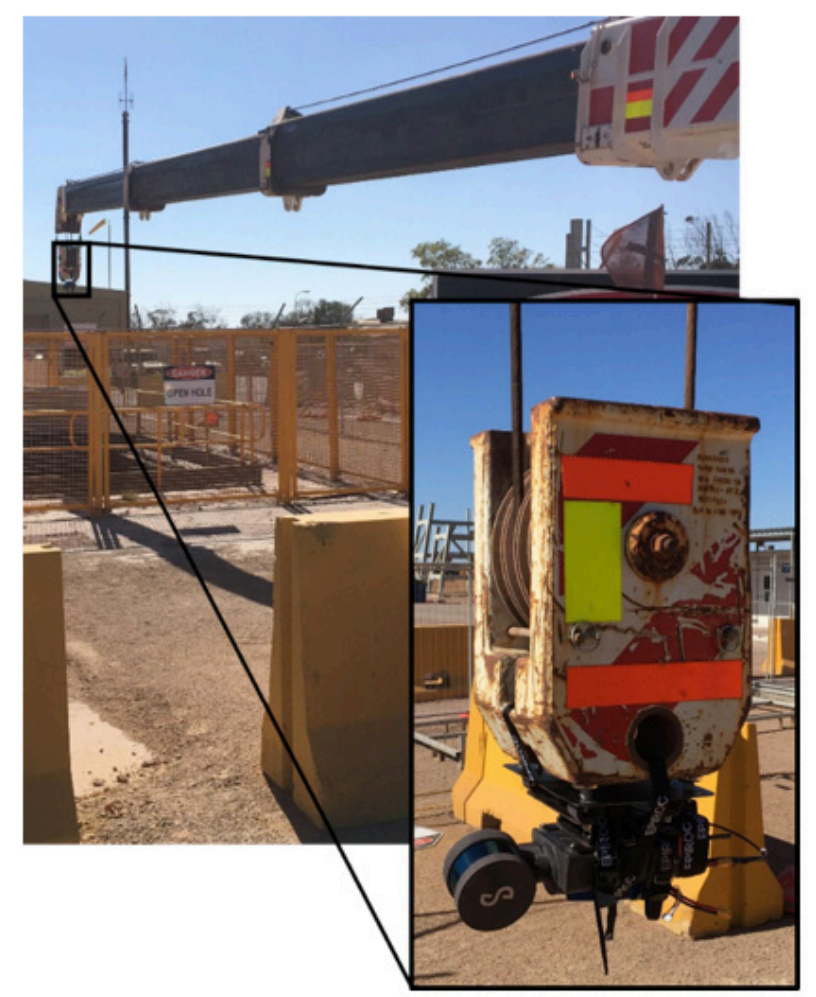

Figure 6 The setup used for positioning the Hovermap scanner on the end of a Franna for lowering down a shaft

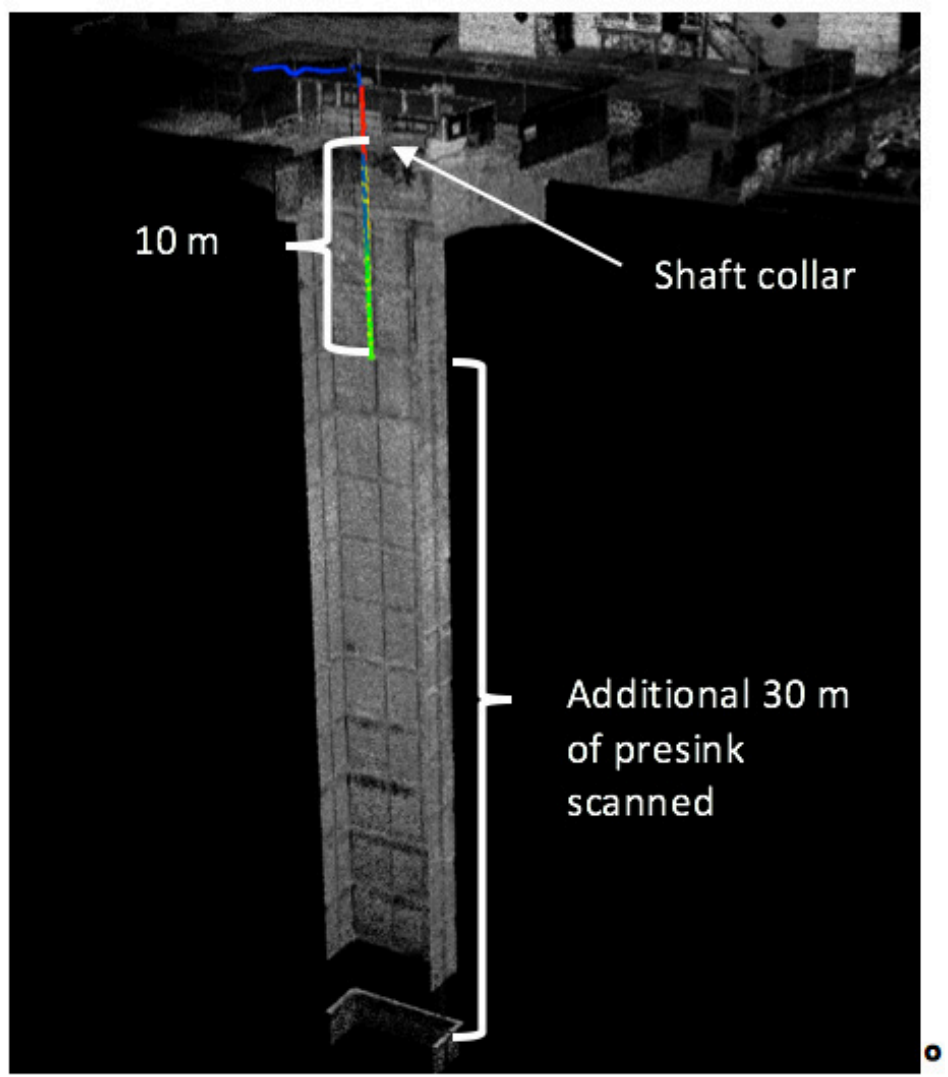

Figure 7 The trajectory of the Hovermap mobile scanner when attached to a Franna and lowered into a shaft for a scan of the presink Colouration refers to time of scan. 


\section{$7 \quad$ Applications within the planning and technical departments}

The Resource Engineering Department at Olympic Dam has been actively pursuing new insights and application for the point clouds produced by the Hovermap scanner. Over the past 12 months, two main uses have been researched; monitoring applications and resource understanding. The following sections provides an overview of the types of applications Hovermap has been used for thus far.

\subsection{Shaft pre-sink deformation monitoring}

Until recently, the main personnel access into the mine was via the original Whenan Shaft. A review of the serviceability of the shaft was undertaken and an upgrade planned. Following the review the shaft was decommissioned as a personal access and from production haulage, with its only purpose now as a ventilation shaft. As part of the decommissioning it was recognised that there are potential instabilities of the initial $40 \mathrm{~m}$ presink located within alluvial sands and sedimentary layers. As a precaution for the identification of an emerging instability, regular monitoring of the presink was introduced, the frequency of which is based on an observational trigger, action, response plan (TARP).

Prior to the decommissioning of the shaft and subsequent removal of all internal infrastructure in 2017, a baseline scan of the presink was conducted using traditional 3-dimensional survey methods, with the scans subsequently stitched together to form a continuous dataset. With the cage since removed, it was asked as to the most appropriate method for continuing the monitoring process, and how best to reproduce a continuous pointcloud data set.

The solution proposed was to attach the Hovermap scanner to a Franna crane located outside the 10 $\mathrm{m}$ exclusion zone as previously described. The scan produced by lowering the Hovermap scanner $10 \mathrm{~m}$ down the shaft produced a pointcloud extending to a depth of $40 \mathrm{~m}$, effectively covering the entire presink.

The main purpose for conducting the scan comparison was to identify any spalling or deflection that might be a precursor for an emerging instability. An external engineering company reviewed the 2019 scan concluding that no curvature or obvious deflection could be observed. Convinced that the analysis could be further than this, two comparison techniques were conducted between the 2017 and 2019 pointclouds.

Prior to the comparison, the pointclouds underwent a QA/QC to confirm there were no erroneous data points that are occasionally found within mobile scanning point clouds using SLAM techniques, or through the process of stitching statics scans together. It was found that the 2019 dataset had some erroneous data captured during the final few seconds of the scans. It was recognised as having poor alignment with the remainder of the scan and were subsequently removed via a time-based filter. LiDAR point clouds, whether static or mobile, will inherently have a thickness associated with them. This thickness depends on a range of factors, but typically high-end static scanners will create scans with less variance. A major factor in thickness is range, the distance from the scanning sensor to the object reflecting the photon. In the case of mobile scanning, this range varies as the object is scanned from various distances. As such, to reduce the thickness a range filter can be applied, removing data points scanned at a greater distance. With all LiDAR scans, this variability in dataset precision must be considered in the final point cloud. In this instance, the points at the base of the presink, will be less precise than those nearer to the collar. As both scans has a point density far exceeding what as required for the comparison methods, both were subsampled to a minimum spacing of $25 \mathrm{~mm}$. This spacing has been found to balance between visualisation continuity and duration required for completing comparison calculations. 
The first technique for change detection used a point-to-point (P2P) distance comparison. This method rapid provides as display of net change between the two point clouds. To distinguish what change has occurred further analysis on the areas of interest is required. For change detection and convergence monitoring, the P2P method is commonly used as a first-pass. The results from this method showed localised changes, typically in the range of $1-10 \mathrm{~cm}$. No larger-scale deflection could be identified between the 2017 and 2019 scans. This result was in agreeance with the external report.

The second method used for change detection used a principal components analysis (PCA). The advantage of using a PCA method is the ability to distinguish convergence from divergence, rather than net-change which is the output from (P2P). The results from the PCA analysis (Figure 8), showed that a vast majority of the presink is showing less than $25 \mathrm{~mm}$ of movement, and there is no flexural change. The minor areas of change were in agreeance with the P2P result, but with the added benefit of confirming negativechange, or divergence. This result, in combination with the vertical pattern, suggested the changes were a result of air and water pipework being removed during the decommissioning.
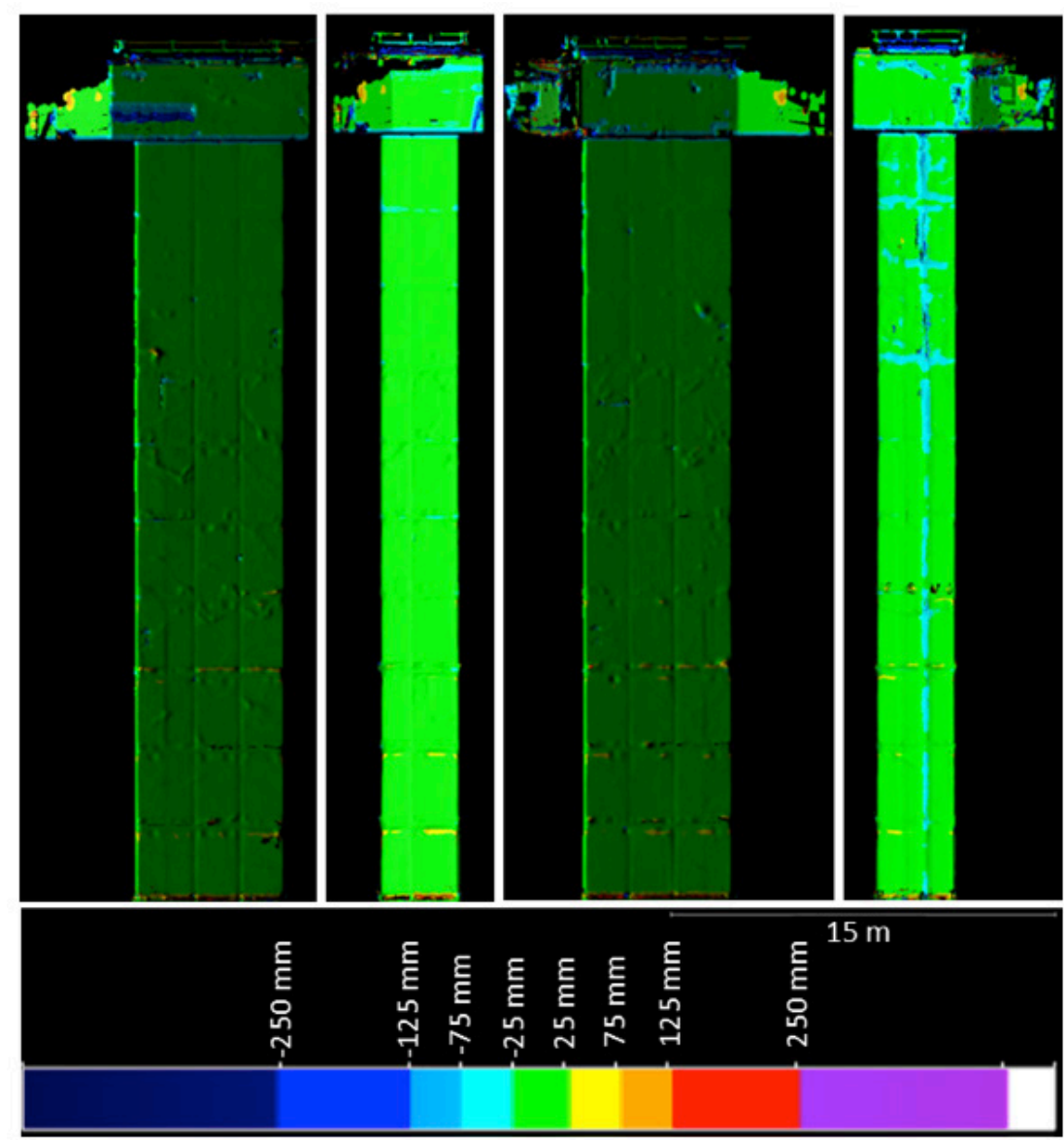

Figure 8 A series of images showing the results of the LiDAR point cloud comparison over a two year period. The shaft lining has remained stable, but minor variation are observed due to the removal of pipework

As per the TARP for monitoring requirements, the next repeat scan is scheduled for late-2020 and this methodology of scanning has been incorporated as an annual process. Olympic Dam is now reviewing the process for the scanning of other vertical infrastructure and excavations using this methodology for spatially-continuous monitoring.

\subsection{Structural mapping and stope design}

The process of structural mapping in underground environments has been limited to either drill holes, (core logging or geophysics methods), or various mapping techniques in tunnel development (i.e. facemapping or window mapping). More recently, the use of LiDAR has shown promise for digitally capturing structural information in development tunnels. The question of persistence of structures has remained 
given the limited exposure to structures in these techniques, often requiring the interpretation of these structures. Within stoping mines, the large voids created would provide Geotechnical Engineers large exposed rock-faces to map if access was possible. Furthermore, these large exposures would provide a greater understanding of persistence and the interaction between discontinuities at greater length-scales.

A common empirical stability method is the Matthews Stope Stability Method (Matthews 1981) and Potvin Modified Stability Assessment (1988), both of which requires the input of number and orientation of joint sets in their assessment. These joint sets are often captured through window mapping in the vicinity of the stope location, but the ability to back calculate whether the joint sets captured through drive mapping or in-fact the same sets controlling stope-scale stability has often been difficult.

High-resolution point clouds from in-stope drone-based LiDAR scans have provided an opportunity for Geotechnical Engineers to review the final stable stope shape and determine the discontinuity sets influencing the final shape (Figure 9-A and -B). This access and new mapping technique allows the back calculation between the drive-scale mapped joint sets and the stope-scale discontinuity sets (Figure 10).
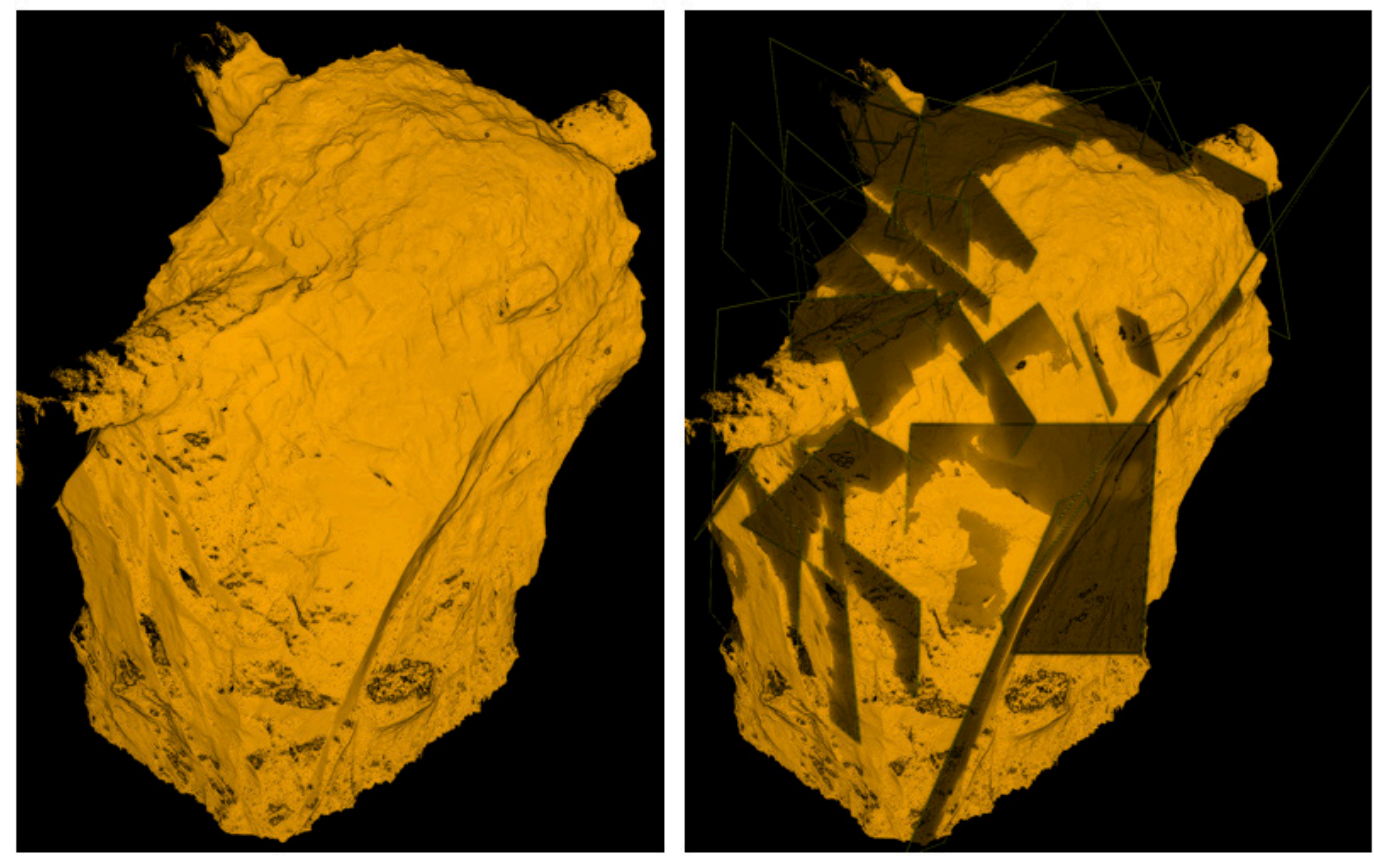

Figure 9 A) The high resolution point cloud results from a drone based scan. B) The discontinuity planar features extracted from the point cloud
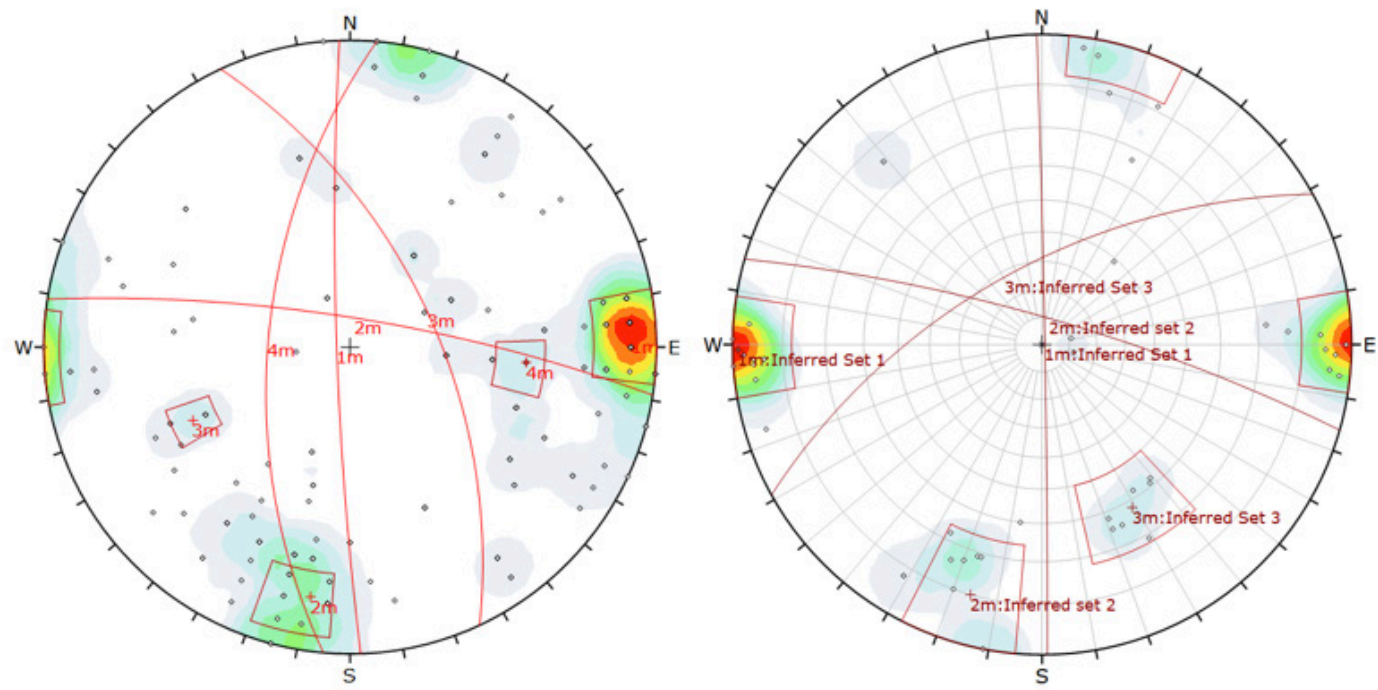

Figure 10 (Left) Steronet composed of structures from window mapping used for the stability assessment. (Right) Steronet composed from structures picked from the final stope shape point cloud 
The result for the stope shown in Figure 10 and the comparison between mapped drive-scale and picked stope-scale structure shows a close correlation of the dip and dip-direction for the two major sets. The scatter of smaller scale structure is much higher within the drive mapping, whereas a clearer third, minor, joint-set is more obvious from the stope scale structure.

This back analysis and comparison between mapped drive-scale structure and LiDAR-based stope-scale structure was undertaken for each of the stopes within one of the mining blocks at Olympic Dam that had drone-based LiDAR data (Figure 11). The results from this back analysis showed that in most cases the major joints sets controlling the final stope shape were identified through by the drive-mapping, but the minor-joint sets were less predictable (Figure 12).

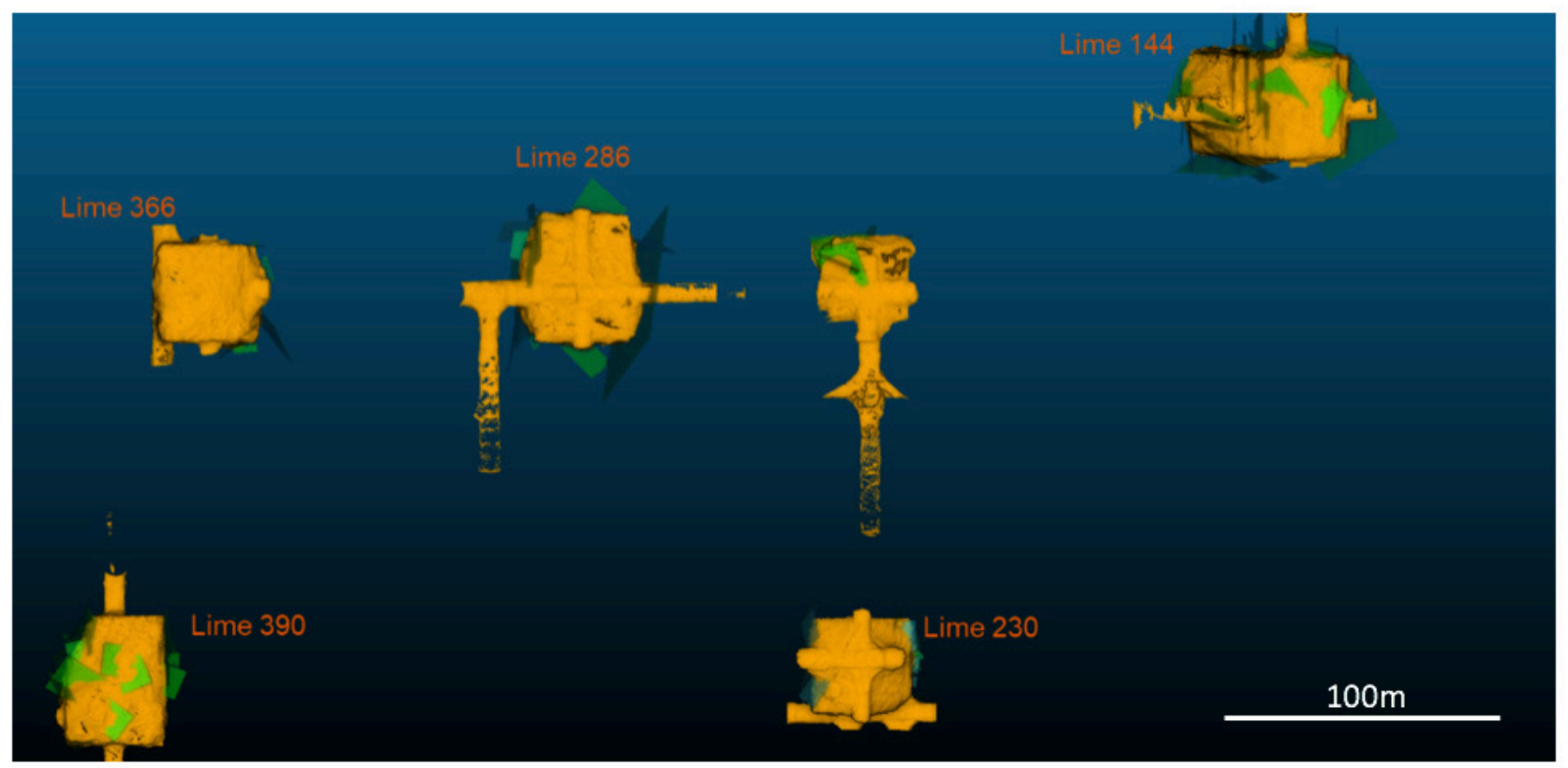

Figure 11 The limes stoping block showing the picked discontinuities throughout
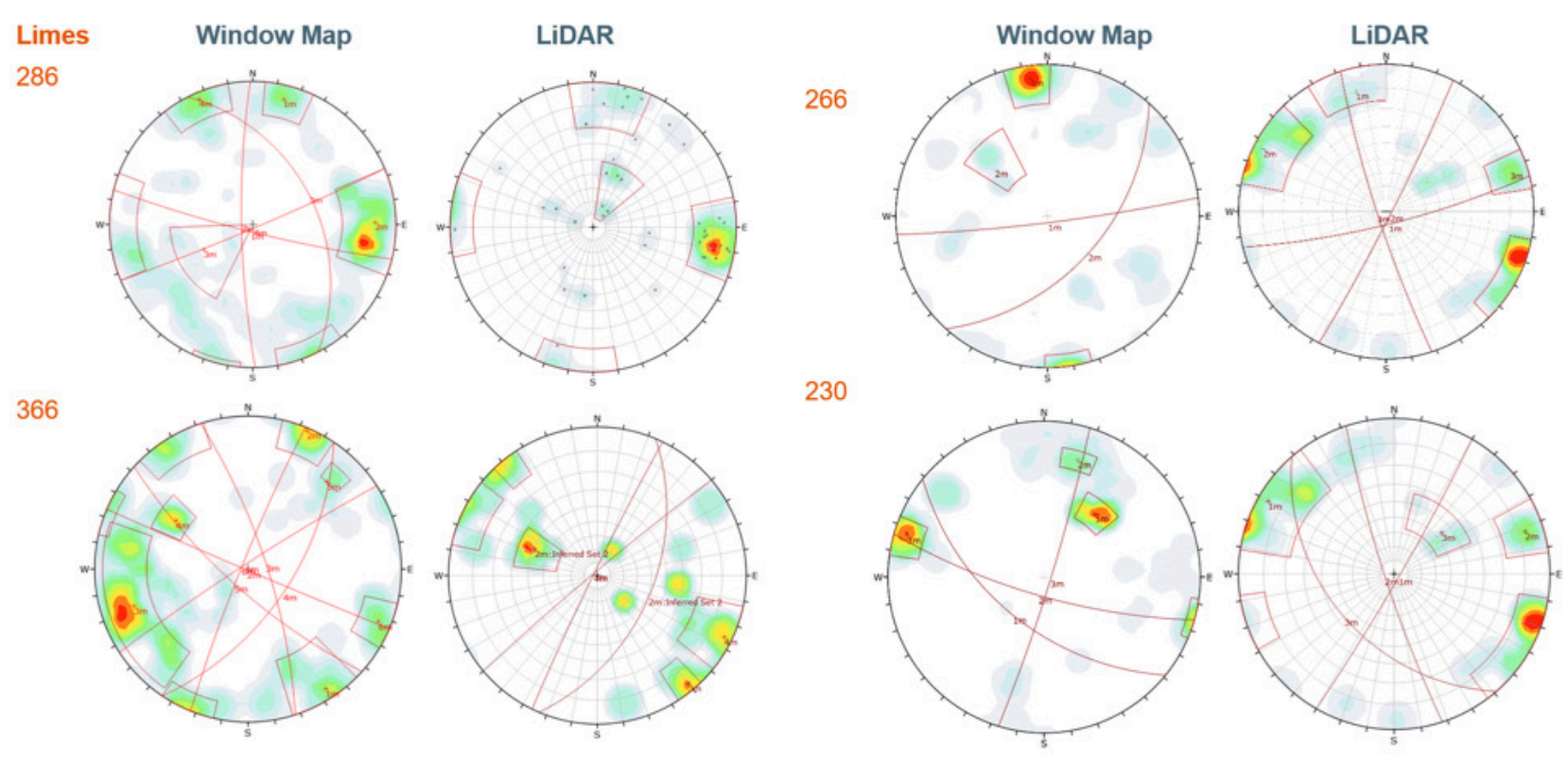

Figure 12 Summary of structural sets from window mapping used in the initial stability assessments and the corresponding LiDAR in-stope picked structures following the final stope scans

The outcome from this has now instigated a review of window mapping procedures to identify the discrepancies and improve the design process. Furthermore, the structural sets picked from the stopes provides additional data for adjacent stopes and their stability assessments. 


\subsection{Geological interpretation of stope scale structures picked from the stope scan}

Geologists at Olympic Dam provide structural data and interpretations to Geotechnical Engineers and Mine Design Engineers in order to estimate the behaviour of the rock mass when it is mined. Geologists are also asked to provide a 3D interpretation of an area that is experiencing problems whilst it is being mined, such as broken ground in development or stopes (Widdup et al. 2004). The integration of high-resolution LiDAR drone scans into existing Mine Geology modelling workflows within Leapfrog GeoTM provides an opportunity to improve critical aspects of the current geological modelling process and will become a key tool in Olympic Dam's continued journey towards best-informed, realistic and stable mine plans involving mapping out and understanding structural complexity. LiDAR will enable increase confidence in reconciliation of our stope-scale structural models that Mine Geology provide to Geotechnical Engineers for stability assessment using a mature workflow and the availability of higher-resolution information will improve understanding of how mine-area scale faults interact with stope-scale discontinuities and their overall impact on stope performance.

For over twenty-five years underground production at Olympic Dam was focussed in the northern mining area (NMA) - a geologically simple orebody, characterised by linear, sub-vertically dipping, structurally focussed hematite-rich breccia bodies (Taylor et al. 2019). During this period WMC Resources and SRK Consulting partnered to refine the current system of structural mapping, and to use historical data (mapping and CMS) to produce a structural interpretation covering a large part of the northern mining area in an effort to understand the influence of structural geology on stope performance (Widdup et al. 2004). The three-dimensional structural interpretation with more than 600 structures of $>50 \mathrm{~m}$ strike length incorporated into the structural framework, which covers a study area $1500 \mathrm{~m} \times 600 \mathrm{~m}$ $\times 350 \mathrm{~m}$ was used as a predictor of primary weaknesses that could be causing over-break and underbreak within existing NMA stopes. In 2015, rapid underground expansion into the geologically complex southern mining area (SMA) drove the requirement for a renewed focus on detailed structural models to support improved mining performance. The interpretation of high-resolution LiDAR scans will continue to build on the good work of Widdup et al. and the three-dimensional structural framework model. LiDAR will enable Mine Geology to accurately identify key structural features, effectively integrating these planar features into existing structural models whilst simultaneously improving the confidence of new interpretations.

There are ultimately three-scales to a structural workflow and focus should be on all three, consistently across the mining footprint (Figure 13). First there are Deposit-scale or major 'first-order' faults controls on the mineralisation footprint and inform the deformation events that shape deposit geometry. Mine Area-scale, which are second to third order faults controlling local-scale orebody geometries and their dislocation, whilst also controlling later remobilisation and/or hydrothermal alteration e.g. indicator alteration. Finally, at the Stope-scale and how do all faults interact at the stope-scale and their local damage zones (Clark 2020).
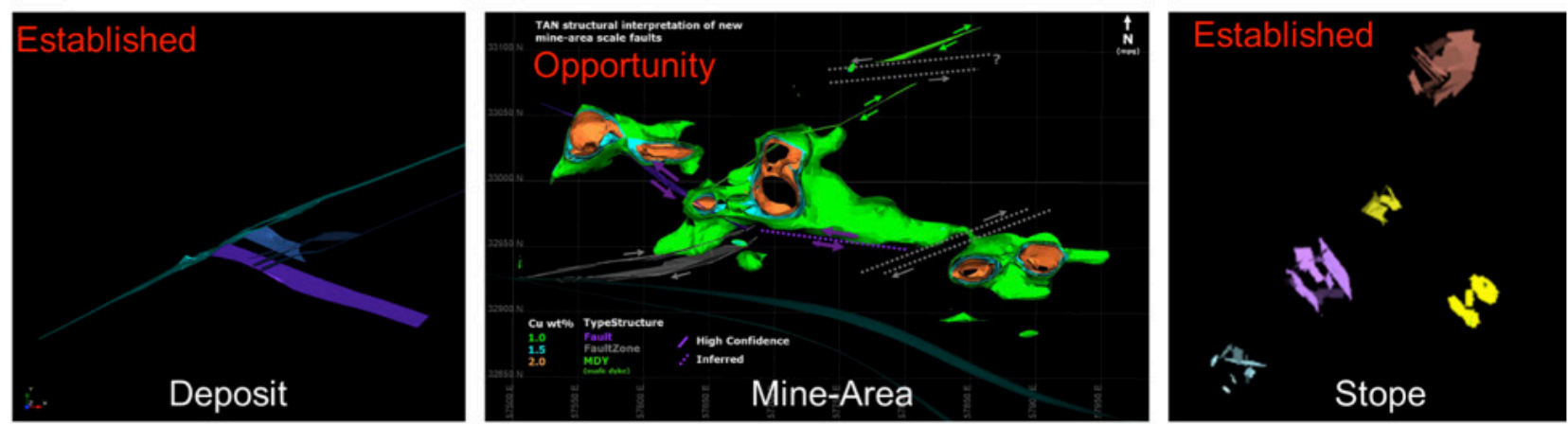

Figure 13 An overview of the three scale of structural modelling at Olympic Dam and the status of modelling workflows; far left - Deposit-scale, middle - Mine Area-scale, right - Stope-Scale. (Clark 2020)

Mine Geology have well established workflows for modelling 'first-order' and stope-scale structures. Deposit-scale structures often manifest as zone of intense alteration (hematite and/or sericite), low RQD 
or offsets in marker lithology's and mineralisation, these are mappable with current data. Stope-scale structures are not necessarily faults, rather any discontinuities and Mine Geology have routinely captured and modelled these discontinuities - that is the one consistent product for the past 20 years and are a requisite input to the initial shape-review process (Figure 14). LiDAR will improve reconciliation confidence of our stope-scale structural models that we provide to the Geotechnical Department, however the usefulness of these structural models is primarily to inform the stope stability assessments and ground support controls on future adjacent stopes and are unlikely to materially inform our shape designs. The danger is always over-interpreting information at this scale, hence it is unlikely the stope shape designs will be materially influenced, rather the stope stability assessments driving changes in drill and blast and ground support design.

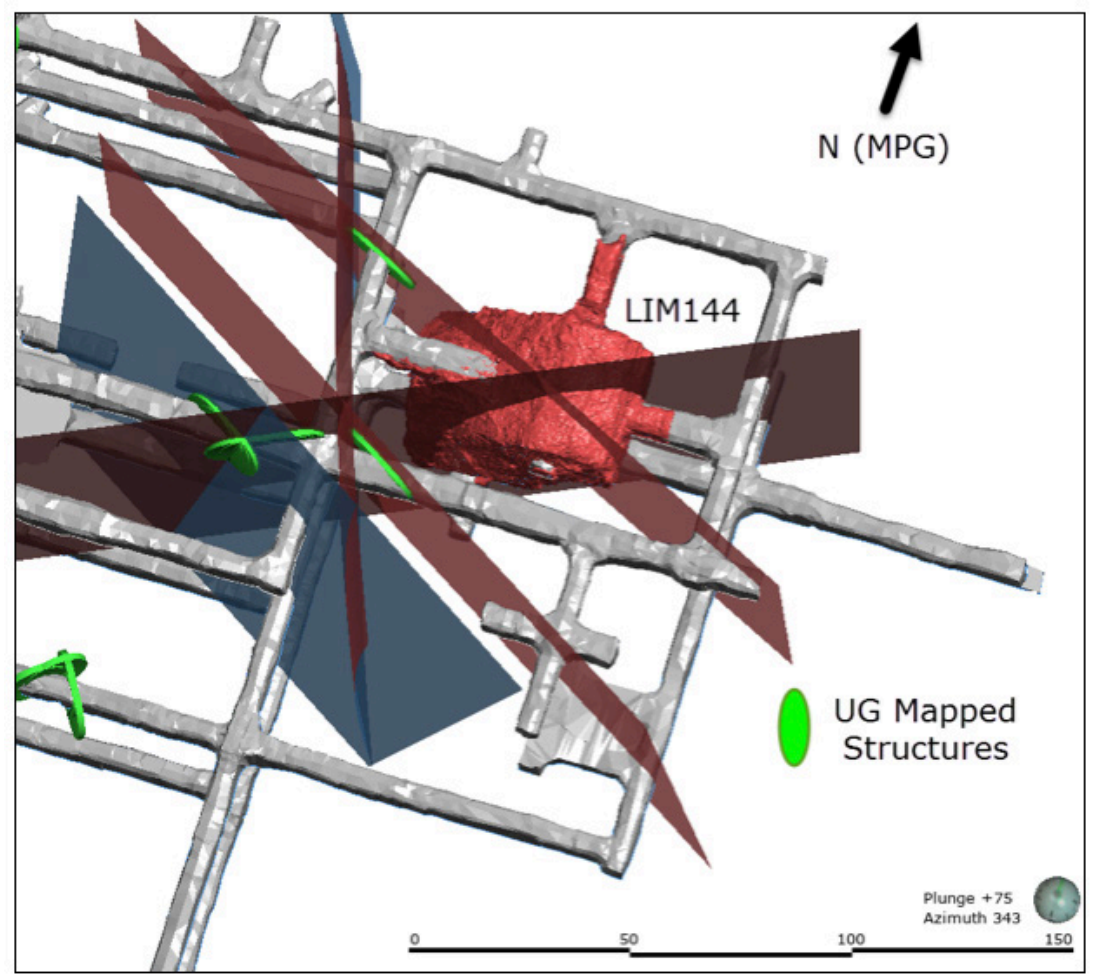

Figure 14 Current methodology for stope scale structural interpretation is converting mapped point observations to structural planes

However a gap exists in effective modelling of mine-area scale structures and as a result Olympic Dam fundamentally lack a detailed Mine-area scale fault model in new mining areas. The key value opportunity is to hone in on important mine-area scale faults and their interaction with stope-scale structures leading to poor performance. Olympic Dam could have been doing this historically with CMS data, however LiDAR offers next-level higher-resolution and will assist with this interpretation. LiDAR will provide another tool to circumnavigate challenges in developing a Mine-Area scale structural model such as; 1) shotcrete to gradeline which reduces visibility of structures in development and results in variable quality and quantity of historic underground mapping, 2) coarse resolution, shadowing and potential for survey artifacts in existing CMS render geological interpretation impractical and 3) low density of orientated underground diamond drillhole data and current structures are modelled using spatial lineaments in logged fault zones with similar infill thickness and type. The successful integration of structural interpretations from LiDAR into current Mine-Area structural models will be key in modernising modelling workflows.

Figures 15-17 below show the proposed workflow for the interpretation of high resolution drone scans on planar discontinuities in Leapfrog Geo using structural discs, which can be rapidly integrated into existing structural models. Successful integration of previously underground mapped data with structural discontinuities identified from the drone scan has significantly improved the accuracy and confidence of Mine-Area scale structures and It will better-inform geotechnical constraints towards a more realistic and safer mining plan. 

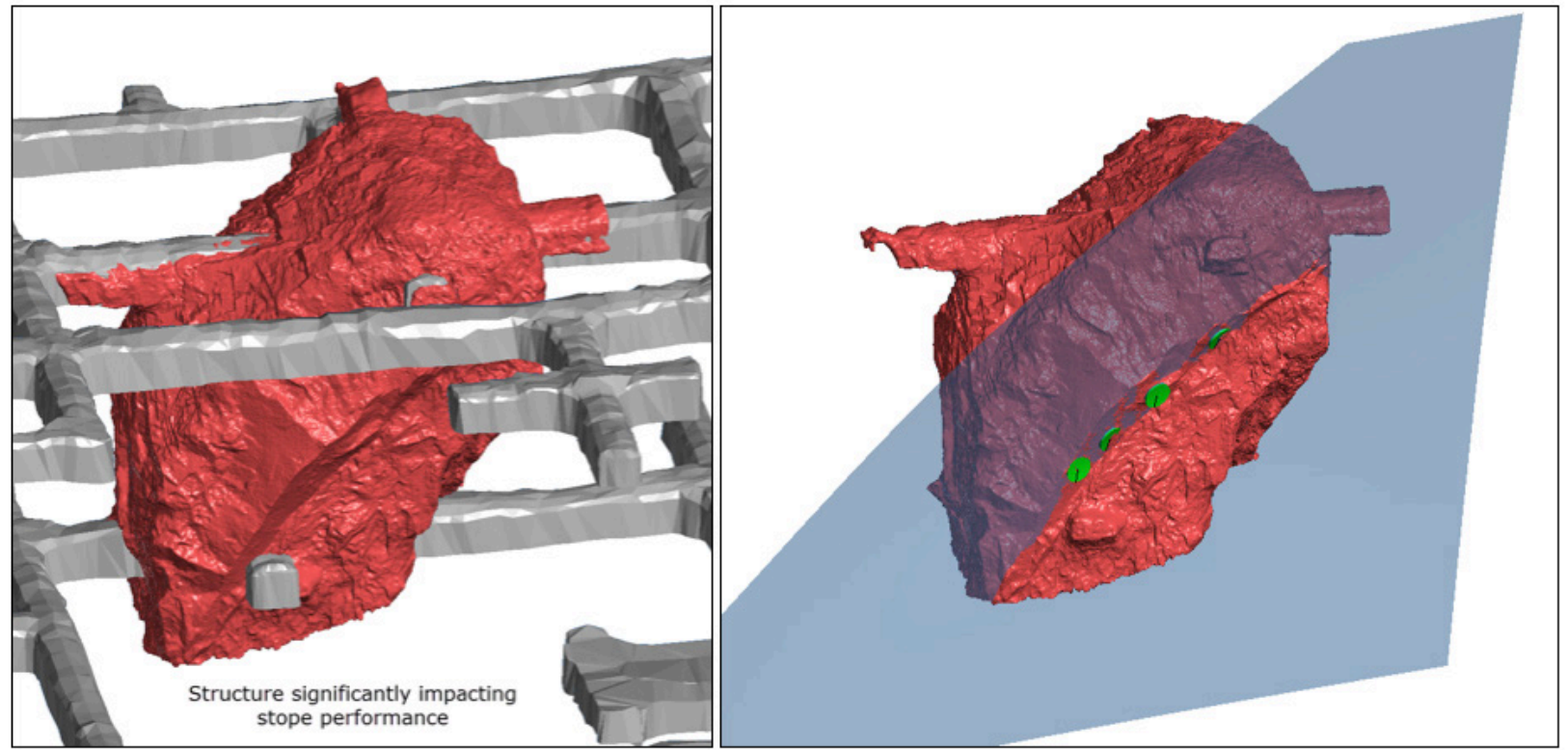

Figure 15 Interpreting high resolution drone scans on planar discontinuities in Leapfrog Geo using structural discs, which can be rapidly integrated into existing Mine-Are scale structural models

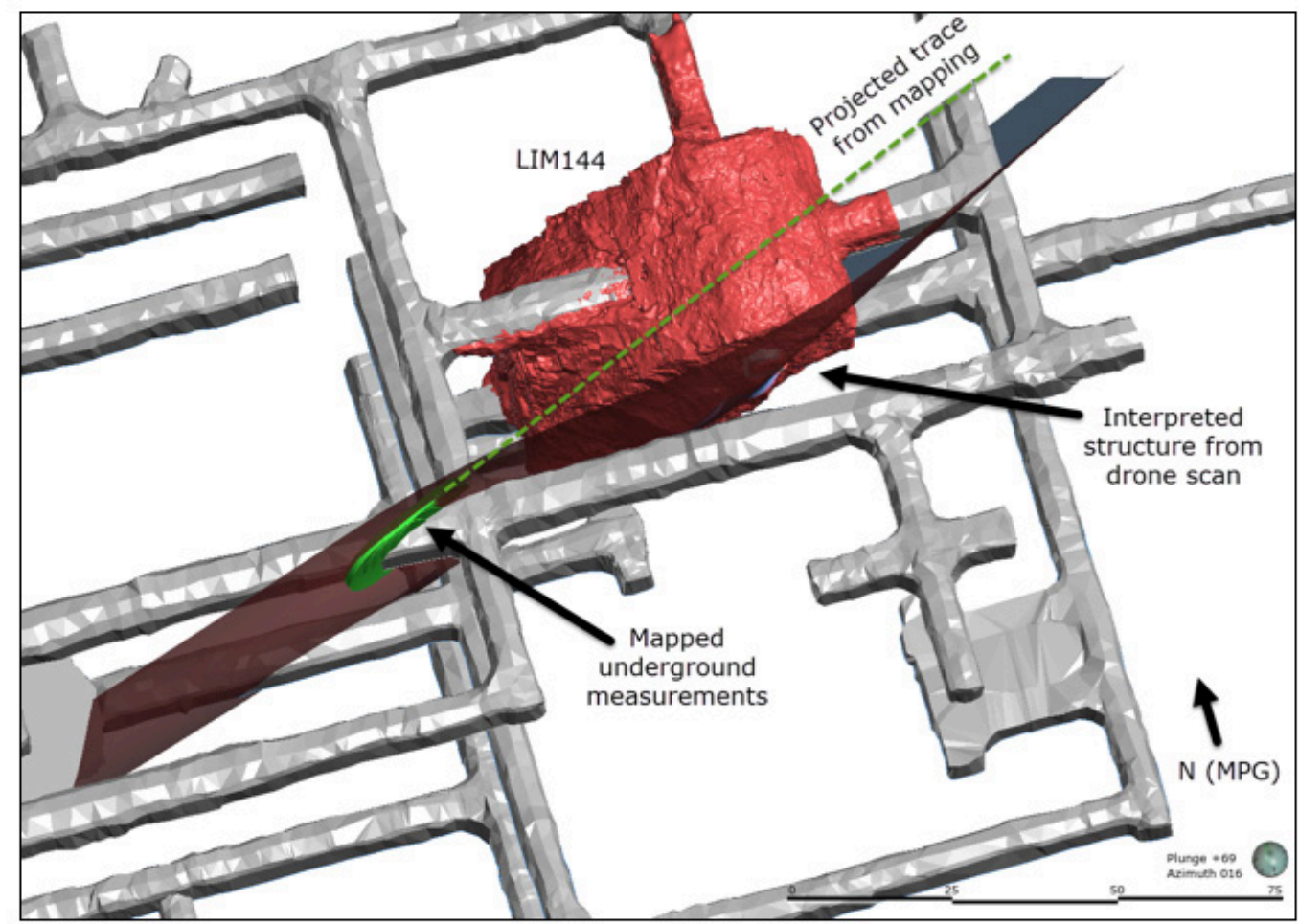

Figure 16 Successful integration of previously underground mapped data with structural discontinuities identified from the drone scan has significantly improved the accuracy and confidence of the MineArea scale structure

Figure 17 highlights the ability to premeditate structures controlling poor ground in future stopes. The true value of the new high-resolution scan will be realised once there is sufficient drone scan density within a mining front that the key structures and structural orientations controlling mining performance become apparent and these can be transposed onto future, adjacent tertiary stopes. This work will enable Mine Geology to better inform geotechnical constraints towards a more realistic and safer mining plan. 


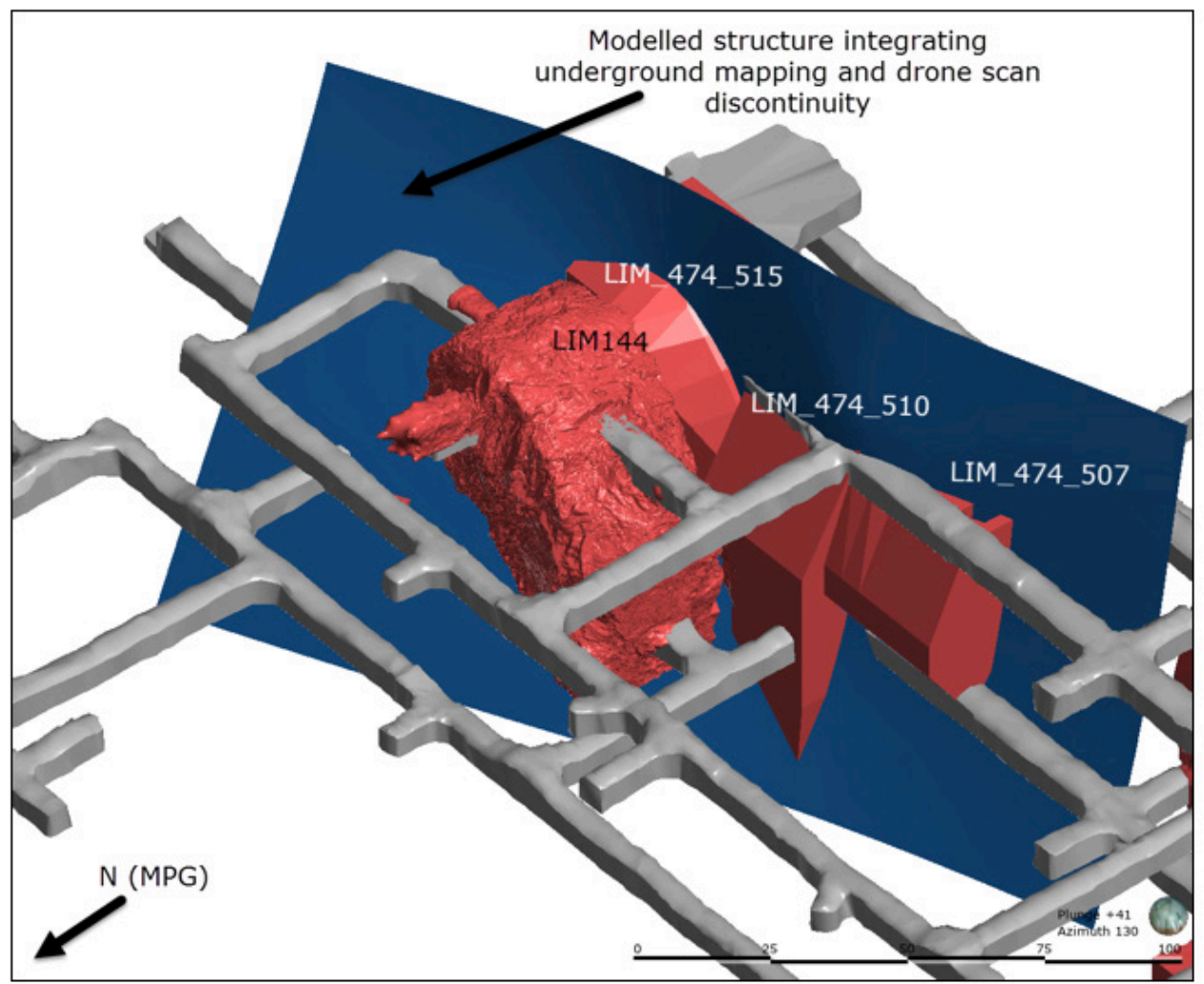

Figure 17 Projection of the modelled discontinuity, controlling major over-break, onto neighbouring planned stopes. This will enable proactive ground-support and mining measures

In summary, LiDAR will also enable increase confidence in reconciliation of our stope-scale structural models that Mine Geology provide to Geotech for stability assessment via 3D validation. In addition, Olympic Dam require a detailed a mine-area scale fault model in our new mining areas, LiDAR may help resolve and/or independently confirm our existing interpretations. The availability of higher-resolution information will improve understanding of how mine-area scale faults interact with stope-scale discontinuities and their overall impact on stope performance.

\section{Conclusions}

The use of new technology in underground mining invariably encounters initial constraints and difficulties prior to full utilisation. However once these difficulties have been overcome, the operational benefits offer new opportunities for mining operations to make significant gains. The technology referenced above was not only responsible for improvements in an existing process, the technology has allowed data to be obtained from sources that were previously unfeasible, unsafe or impractical. Operator safety has improved, while improving data volume and quality.

The benefits of this system have benefitted the operation as a whole, not only the implementing department. As shown the system has improved operational processes, but has also provided data for technical analysis and asset project work.

It should be a key consideration for any operation looking at new technology to look beyond the process they are looking to improve to areas where the outputs of technology can be utilised. 


\section{Acknowledgement}

The authors would like to acknowledge the contribution of Emesent Pty for all their continual contribution to the project at Olympic Dam.

The authors would also like to acknowledge everyone at BHP Olympic Dam who have been involved in this project, particularly the Drone Pilot Team whose continual hard work have made this project a continual success.

Finally, the authors would like to acknowledge BHP, who approved the publishing of this paper.

\section{References}

Clark, J 2020, 'Geotech teaching talk—Leapfrog update \& geological insights', BHP. Roxby Downs - Olympic Dam, Internal publication.

Jones, E, Reardon, D \& Hrabar, S 2019. 'The use of automated drones in underground hard rock mines', Proc. Future Mining 2019, The Australasian Institute of Mining and Metallurgy, Melbourne, pp. 34-46.

Matthews, KE, Hoek, E, Wyllie, DC \& Stewart, S 1981, 'Prediction of stable excavation spans for mining at depths below 1,000 metres in hard rock', Golder Associates, CANMET Library \& Documentation Services Division, Vancouver.

Potvin, Y 1988, 'Empirical open stope design in Canada' PhD thesis, University of British Columbia.

Taylor, JR, Clark, JM \& Levy, I. 2019. 'BHP Olympic Dam's Transformation to Live Implicit Modelling and Innovative Solutions to Working in Data Dense Environments', Proc. Mining Geology Conference, Perth, 25-26 November, 2019, The Australasian Institute of Mining and Metallurgy: Melbourne.

Widdup H, Fouet T, Hodgkison J, McCuaig T C \& Miller J 2004, 'A three dimensional structural interpretation of the Olympic Dam deposit - Implications for mine planning and exploration', Hi Tech and World Competitive Mineral Success Stories Around the Pacific Rim, Proc. Pacrim 2004 Conference, Adelaide, 19-22 September, 2004, The Australasian Institute of Mining and Metallurgy: Melbourne, pp. 417-426 